\title{
Multiscale Simulation of Electronic and Optoelectronic Devices with TiberCAD
}

\author{
M. Auf der Maur, M. Povolotskyi, F. Sacconi, G. Romano, \\ E. Petrolati, and A. Di Carlo \\ OLAB, Department of Electronic Engineering, \\ University of Rome "Tor Vergata", Via del Politecnico 1, 00133 Rome, Italy \\ \{auf.der.maur|povolotskyi|dicarlo\}@ing.uniroma2.it
}

\begin{abstract}
We present the TIBERCAD multiscale device simulation software. The scope of the project is a full description of charge transport and optoelectronic properties of devices with embedded active regions of nanometer-scale. We show simulations of a GaN LED that requires modeling of strain, transport of electrons, holes and excitons and device heating.
\end{abstract}

\section{The TIBERCAD Device Simulator}

The TIBERCAD project is aimed at the implementation of a device simulator which captures the most important physical concepts encountered in present and emerging electronic and opto-electronic devices. On the one hand the down-scaling of device dimensions requires the inclusion of more advanced quantum mechanical concepts which go beyond classical transport theories. On the other hand, functionality of new emerging devices is based both on electrons/holes, and other quasi-particles such as excitons, polaritons, etc. Usually the active part of a device which needs a more elaborate and careful treatment is small compared to the overall simulation domain. The computational cost of the more accurate model however forbids its application to the whole domain. In order to solve these problems, a multiscale simulation software is needed [1].

\section{Implemented Physical Models}

The different physical models implemented in TIBERCAD are described in the following sections. All models are currently implemented for the stationary case only. The discretization of the governing equations is done using the finite element method [2]. The different physical models need not be simulated all in the same space domain but can be restricted to the domain of interest.

\subsection{Strain}

TIBERCAD implements a structural model that allows to calculate strain and shape deformation of lattice mismatched heterostructures. It is based on linear elasticity theory of solids that assumes a pseudomorphic interface between different materials [3]. Ex- 
ternal mechanical forces can be included in the simulation. As a result we obtain the strain map, the shape of the deformed structure and the piezoelectric polarization.

\subsection{Quantum-mechanical Models}

Quantum-mechanical models are needed to calculate the eigenstates of confined particles. The models implemented in TiBERCAD are based on the envelope function approximation including single-band and multiband $\mathbf{k} \mathbf{p}$ approaches. A stationary Schrödinger equation is solved to obtain the energy spectrum, particle density and the probabilities of optical transitions [4]. The quantum-mechanical models are also used to calculate the valence and conduction band parameters in presence of strain.

\subsection{Semi-classical Transport}

Transport of quasi-particles is modeled based on semi-classical theory. The particle flux is assumed to be equal to the gradient of a driving potential multiplied by a particle conductivity $\mathbf{j}=-\sigma \nabla \phi$. In the case of electrons and holes the driving potential is the respective electro-chemical potential and the conductivity equals $\mu_{n} n$ and $\mu_{p} p$, respectively, where $\mu_{n}, \mu_{p}$ and $n, p$ are the mobilities and the particle densities. This model is connected to the drift-diffusion approximation by means of the Einsteinrelation $D=k_{B} T / e$, where $D$ is the diffusivity. In nonisothermal simulations the Seebeck effect is included by writing the particle flux as $\mathbf{j}=-\sigma \nabla(\phi+P \nabla T)$, where $P$ is the absolut thermoelectric power [5].

\subsection{Thermal Transport}

Modeling of thermal transport is based on a thermodynamic model [5]. Self-heating due to Joule and Thomson-Peltier effect and generation/recombination phenomena is considered. The thermal conductivity is assumed to be temperature dependent which leads to a nonlinear equation for the heat diffusion.

\subsection{Atomistic Modeling}

Small, but important regions of a device can be described atomistically based on empirical and semiempirical models, including structural, electronic and thermal properties [6]. The models are based on tight-binding and density functional theory and will be extended to Green's functions theory for atomistic description of transport.

\section{Simulation Examples}

As first example we present simulation results of a GaN pin-diode with embedded $\mathrm{In}_{0.05} \mathrm{Ga}_{0.95} \mathrm{~N}$ QW. Such a structure is proposed as a realization of a polariton laser [7]. The computational cost is reduced by one half exploiting the device symmetry with respect to the $x$-coordinate. The heat transport is solved over a $0.5 \mathrm{~mm} \times 0.8 \mathrm{~mm}$ size region that includes the GaN substrate, the heterostructure and the air around the device, whereas the particle transport simulation is limited to a part of the device of 
$0.5 \mu \mathrm{m} \times 0.2 \mathrm{~mm}$ size, where current flow is expected. In Fig. 1 we show the temperature map at a bias voltage of $3.4 \mathrm{~V}$, which corresponds to a current of approximately $1 \mathrm{~A} / \mathrm{cm}$. A temperature increase of about $5 \mathrm{~K}$ can be observed at the center of the device. Fig. 2 presents the exciton density in the InGaN well and several streamlines of the electrical current.

As second example we show simulation results for an $\mathrm{AlGaN} / \mathrm{GaN}$ nanocolumn pindiode of $20 \mathrm{~nm}$ lateral size and $200 \mathrm{~nm}$ height under forward bias of $4.5 \mathrm{~V}$ (fig. 3). In such structures one observes current confinement due to inhomogeneous strain and related piezoelectric effect.

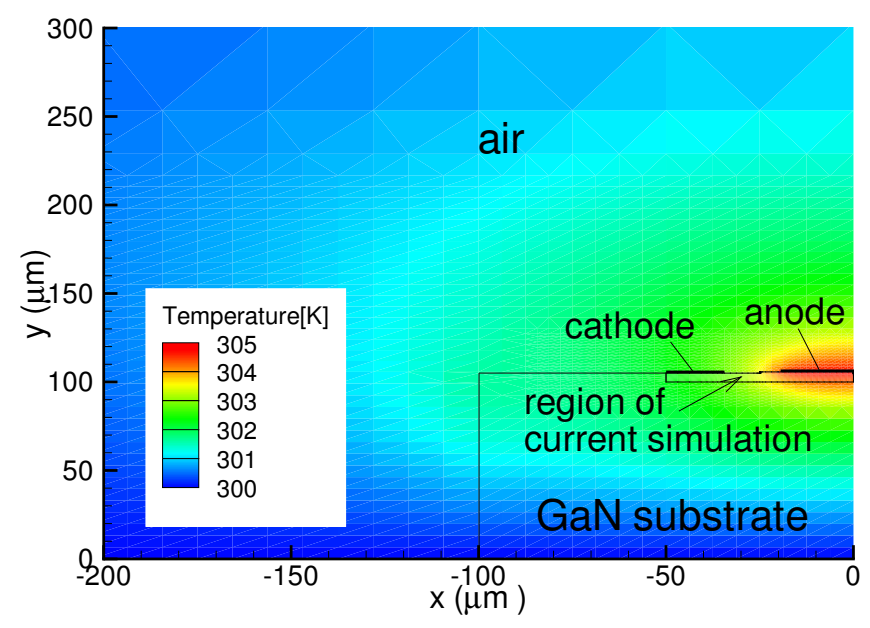

Figure 1: Temperature map at a bias voltage of $3.4 \mathrm{~V}$.

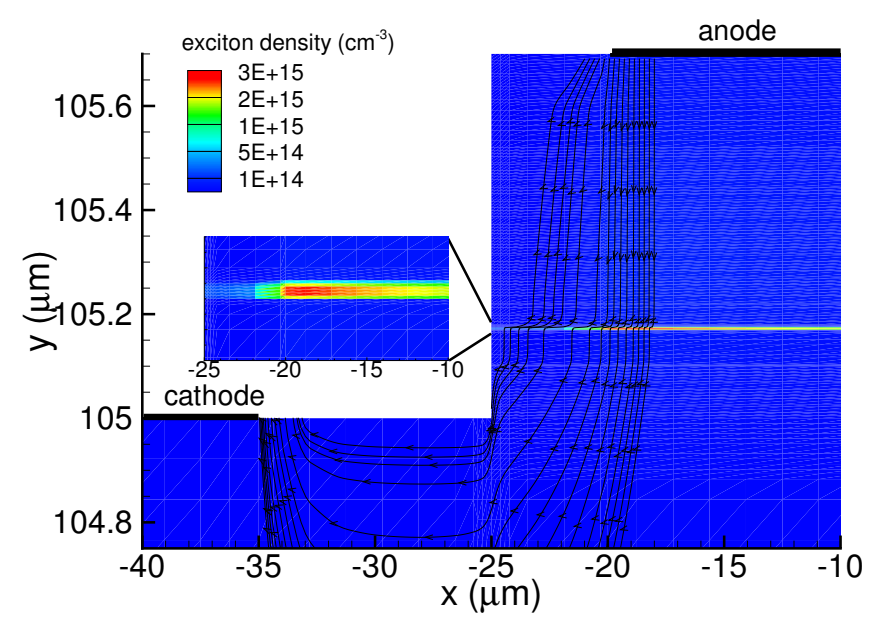

Figure 2: Streamlines of electrical current and exciton density at a bias voltage of $3.4 \mathrm{~V}$. 


\section{Electron current density}

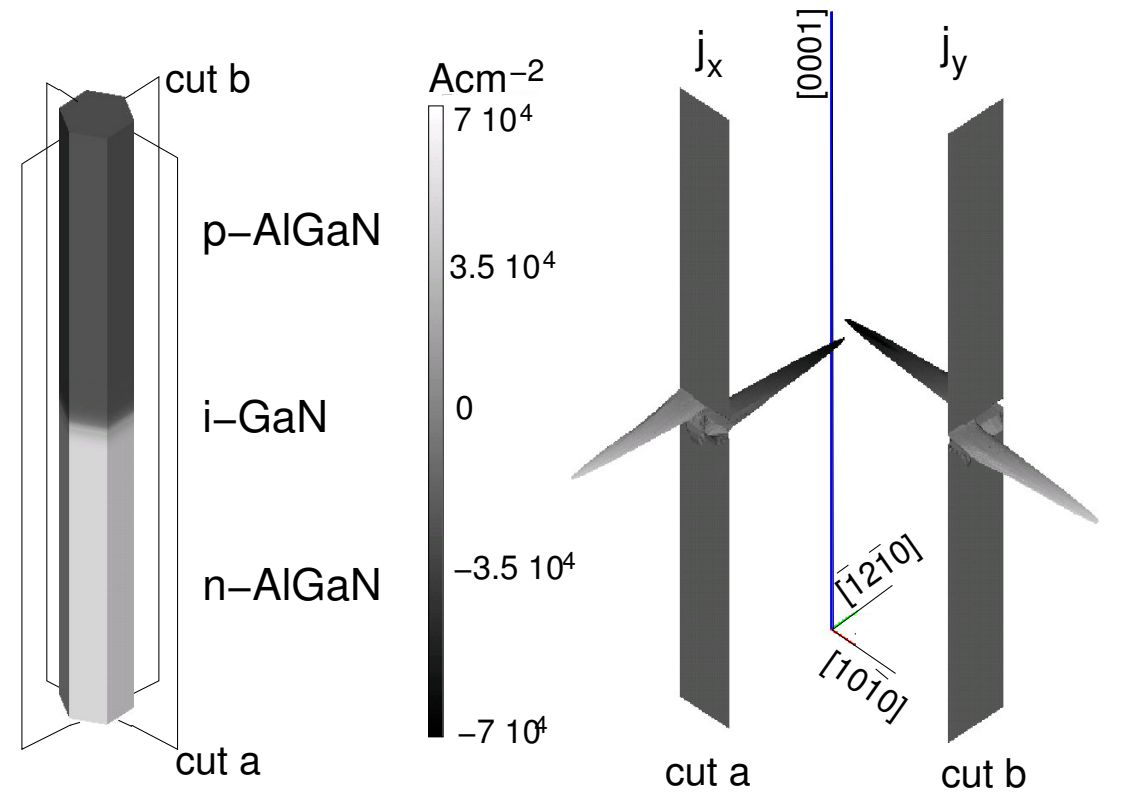

Figure 3: In-plane components of the electron current density inside the intrinsic GaN region of an $\mathrm{AlGaN} / \mathrm{GaN}$ nanocolumn pin-diode over two perpendicular cutplanes.

\section{Acknowledgements}

We acknolwdge the EU FET-Open Program, Stimscat FP6-517769, for financial support.

\section{References}

[1] TiberCAD simulation package, http://www.tibercad.org.

[2] B. S. Kirk and J. W. Peterson, libMesh library, http://libmesh. sourceforge org.

[3] M. Povolotskyi and A. D. Carlo, "Elasticity theory of pseudomorphic heterostructures grown on substratesof arbitrary thickness," J. Appl. Phys. 100, 063514 (2006).

[4] S. L. Chuang, Physics of optoelectronic devices, Wiley Series in Pure and Applied Optics 1st edition (1995).

[5] G. K. Wachutka, "Rigorous Thermodynamic Treatment of Heat Generation and Conduction in Semiconductor Device Modeling," IEEE Transactions on Computer-Aided Design 11, 1141 (1990).

[6] A. Di Carlo, Introducing Molecular Electronics, Springer, Heidelberg (2005).

[7] G. Malpuech et al., "Room-temperature polariton lasers based on GaN microcavities," Appl. Phys. Lett 81, 412 (2002). 